\title{
A Prospective Randomized Comparative Study of Monopolar and Bipolar Transurethral Resection of the Prostate and Photoselective Vaporization of the Prostate in Patients Who Present with Benign Prostatic Obstruction: A Single Center Experience
}

\author{
Anup Kumar, MD, MCh, Pawan Vasudeva, MD, MCh, Niraj Kumar, MD, Biswajit Nanda, MD, \\ Sanjeev K. Jha, MD, and Nayhan Mohanty, MD, MCh
}

\begin{abstract}
Purpose: The first prospective randomized study to compare the safety and short-term efficacy of monopolar transurethral resection of the prostate (TURP), bipolar TURP, and photoselective vaporization of the prostate (PVP) using GreenLight high-performance system $120 \mathrm{~W}$ laser in patients who presented with benign prostatic obstruction (BPO). Patients and Methods: The 186 consecutive patients who presented with BPO and who were planned for surgery were randomized into three groups: Group A, patients who underwent monopolar TURP; group B, patients who underwent bipolar TURP; and group C, patients who underwent PVP. All three groups were subdivided into two subgroups based on prostate volume: Subgroup $1>20 \mathrm{cc}$ and $<50 \mathrm{cc}$, and subgroup 2, between 50 and $80 \mathrm{cc}$. Patients preoperative, perioperative, and follow-up data were recorded and analyzed.

Results: The baseline characteristics of the three groups and subgroups 1 and 2 were comparable. The number of patients in whom postoperative irrigation was instituted, amount of fluid used for postoperative irrigation, duration of postoperative irrigation, postoperative hemoglobin concentration, and duration of catheterization were significantly in favor of group C patients except for the mean operative time, which was significantly longer among them. All three groups demonstrated an increase in International Prostate Symptom Score, quality of life score, and maximum flow rate and decrease in prostate volume and postvoid residual urine at 12-month follow-up. The mean Intgernational Index of Erectile Function-5 score did not show improvement in any group. The need for blood transfusion and clot retention necessitating intervention were significantly lower among group C patients compared with group A, whereas these values for group B patients did not reach significant level compared with either group A or C. These complications were comparable among subgroup 1 patients, whereas subgroup 2 patients had shown results in favor of subgroup C2.

Conclusion: Monopolar TURP, bipolar TURP, and PVP are equally efficacious at 12-month follow-up. PVP has added advantages of lesser blood loss, lesser need for blood transfusion (especially for prostate volume 50-80 cc), and shorter catheterization time.
\end{abstract}

\section{Introduction}

L OWER URINARY TRACT SYMPTOMS (LUTS) that are secondary to benign prostatic enlargement (BPE) represent one of the most common illnesses in aging men. Various treatment modalities are available with a goal to improve quality of life (QoL) and symptoms safely with minimum side effects. ${ }^{1}$ Monopolar transurethral resection of the prostate
(TURP) is still considered the surgical gold standard for the management of symptomatic BPE in prostates between 30 and $80 \mathrm{cc}^{2,3}$ It provides effective and substantial relief of LUTS secondary to BPE, but despite various technical advancements, it has its own disadvantages. It still has a morbidity rate of $11 \%$ and a mortality rate of $0.1 \%$, so the search for an improved and safer technique but with comparable functional results is going on. ${ }^{4}$

Department of Urology, VMMC and Safdarjung Hospital, New Delhi, India. 
The standard monopolar TURP is now challenged by bipolar TURP, with efficacy comparable to it and fewer perioperative complications-negligible TUR syndromes, less blood loss, earlier removal of the catheter, etc. ${ }^{5-7}$ Saline irrigation allows longer operative time leading to safe resection of a larger prostate. ${ }^{8}$ Photoselective vaporization of the prostate (PVP) by GreenLight laser, which provides real-time tissue ablation with excellent hemostasis, even in high-risk patients, has started to gain increasing acceptance. PVP has efficacy comparable to that of TURP, but the main disadvantage is that it does not provide tissue for histologic examination. $^{9-13}$

The lack of a prospective randomized study comparing monopolar TURP, bipolar TURP, and PVP by GreenLight laser prompted us to plan a three-arm study with a focus on perioperative and postoperative complications and shortterm functional results. This three-arm study comparing monopolar TURP, bipolar TURP, and potassium titanyl phosphate laser PVP is the first prospective randomized study to our knowledge after the best possible Medline search.

\section{Patients and Methods}

The study protocol and all procedures were approved by the Institutional Ethics Committee. Consecutive patients attending the urology outpatient department for the period between March 2011 and February 2012 with LUTS secondary to BPE, who were planned for surgery according to the International BPE guidelines of the American Urological Association, and who satisfied the eligibility criteria (inclusion/ exclusion criteria) were included in the study. Inclusion criteria were: (1) Age $\geq 50$ years, (2) International Prostate Symptom Score (IPSS) $>7$, (3) prostate volume measured by transrectal ultrasound (TRUS): $>20$ and $<80 \mathrm{cc}$, (4) maximum flow rate (Qmax) $<15 \mathrm{~mL} / \mathrm{sec}$. Exclusion criteria were: (1) History of prostate, bladder, or urethral surgery, (2) history of spinal surgery or spinal trauma, (3) neurologic disease, (4) postvoid residual urine (PVRU) $>300 \mathrm{~mL}$, (5) indwelling Foley catheter, where indication for catheterization was chronic retention (PVRU >300 mL), (6) a diagnosis of carcinoma of the prostate, carcinoma of the bladder, urethral stricture, (7) patients receiving antiplatelet drugs in whom drugs could not be safely stopped perioperatively, (8) patients who did not give written informed consent.

Initial evaluation included: (1) A detailed clinical historyage, IPSS, International Index of Erectile Function (IIEF-5) score and QoL score; (2) physical examination including examination of external genitalia, digital rectal examination, and focused neurologic examination; (3) investigationsurinalysis, hemoglobin level, serum prostate-specific antigen (PSA) level, prostate volume measurement by TRUS, PVRU estimation by abdominal ultrasound, and Qmax on uroflowmetry (UFR). PVRU and UFR estimation were not performed in patients with an indwelling Foley catheter. In patients with abnormal digital rectal examination and/or PSA level $>4 \mathrm{ng} / \mathrm{mL}$, prostate cancer was ruled out by 12-core TRUS-guided prostate biopsy.

Eligible patients were randomized into three groups using a computer generated randomization table of equal numbers: Group A, patients who underwent monopolar TURP; group $\mathrm{B}$, patients who underwent bipolar TURP; group C, patients who underwent PVP by GreenLight HPS ${ }^{\circledR}$ (high-performance system) $120 \mathrm{~W}$ laser. Then each group was subdivided into two subgroups based on prostate volume (nonrandomized): Subgroup 1, patients with a prostate volume $>20 \mathrm{cc}$ and $<50$ cc; subgroup 2, patients with aprostate volume between 50 and $80 \mathrm{cc}$.

\section{Operative procedure}

Procedures were performed by one of the three experienced consultant urologists in our department, all of whom are well trained with the technique of monopolar TURP, bipolar TURP, and PVP by GreenLight HPS 120W laser.

Group A: Monopolar TURP was performed using a 26F continuous flow resectoscope. 30-degree lens, standard tungsten cutting wire loop, and 1.5\% glycine as irrigant. Power setting used was $160 \mathrm{~W}$ for cutting and $80 \mathrm{~W}$ for coagulation. The resection was carried down to the surgical capsule with the distal limit being the verumontanum.

Group B: Bipolar TURP was performed using a 26F continuous flow resectoscope. The Gyrus ACMI plasmakinetic system was used for the procedure. A 30-degree lens and $0.9 \%$ saline as irrigant were used for the procedure. Power settings used were $180 \mathrm{~W}$ for cutting and $90 \mathrm{~W}$ for coagulation. As with monopolar TURP, the resection was carried down to the surgical capsule with the distal limit being the verumontanum.

Group C: For PVP by GreenLight laser, a 23F continuous flow laserscope, 30-degree lens, $0.9 \%$ sodium chloride as irrigant, and a 600 micron, 70-degree side firing GreenLight laser fiber having a wavelength of $532 \mathrm{~nm}$ was used. Prostatic tissue was vaporized up to the surgical capsule with the distal limit being the verumontanum until a wide TURPlike cavity was made and an unobstructed view of the bladder from the verumontanum was achieved. For vaporization, the laser fiber was moved in a "paint brush fashion" keeping the fiber in close contact with the prostatic tissue. Coagulation, if required, was achieved by the power setting of $30 \mathrm{~W}$.

A three-way indwelling 20F Foley catheter was inserted into the bladder at the end of the procedure in all patients. Postoperative bladder irrigation, if needed, was started with $0.9 \%$ physiologic saline until the urine was sufficiently clear. As an institutional policy, the catheter was removed 24 hours after the irrigation (if started) was stopped and the urine was sufficiently clear. Patients, who failed a trial without the catheter, were recatheterized, and a voiding trial was repeated after 5 days. For all patients, an intravenous antibiotic was administered at induction, and an oral antibiotic was continued for 5 days postcatheter removal.

Perioperative parameters recorded were operative time (the amount of time the resectoscope/laserscope was inside the urethra), amount of fluid used for intraoperative irrigation, number of patients in whom postoperative irrigation was instituted, amount of fluid used for postoperative irrigation, duration of postoperative irrigation, postoperative hemoglobin concentration (morning after surgery), and duration of catheterization (time to first trial without catheter). Patients were followed up in the urology outpatient department at 1, 3, 6, and 12 months. IPSS, QoL score, IIEF 5 score, Qmax, PVRU, residual prostate volume (assessed by transrectal ultrasound), and complications, if any, were recorded at each follow-up visit. 
Primary outcome measures for analysis were:

A. Objective (prostate volume, PVRU, and Qmax).

B. Subjective (IPSS, IIEF5, QoL) parameters.

Secondary outcome measures for analysis were:

A. Perioperative parameters.

B. Complications, if any.

\section{Statistical analysis}

The study parameters were arranged on a Microsoft Excel spreadsheet (Microsoft, Seattle, WA), and SPSS version 12.0 (SPSS Inc., Chicago, IL) software package was used for analysis as follows: (1) A vs B vs C; (2) A1 vs B1 vs C1; and (3) A2 vs B2 vs C2. The parametric outcomes were expressed as mean \pm standard deviation of the group. One way analysis of variance was used for continuous variables, and the chisquare and Fisher exact tests were used to analyze categorical data. Results with a $P$ value $<0.05$ were considered statistically significant.

\section{Results}

A total of 201 patients were screened, of whom 186 patients were found eligible and were randomized -62 patients in each of groups A, B, and C. Subsequent evaluation excluded 2, 5 , and 4 patients from groups $\mathrm{A}, \mathrm{B}$, and $\mathrm{C}$, respectively, leaving behind 60,57 , and 58 patients in the respective groups for analysis. There were 29,30 , and 27 patients in subgroups A1, B1, and C1, whereas subgroups A2, B2, and C2 had 31, 27, and 31 patients, respectively (Fig. 1).

The baseline characteristics of the three groups are summarized in Table 1 . The mean age, preoperative hemoglobin concentration, IPSS score, serum PSA level, Qmax, prostate volume, IIEF-5 score, QoL score, and PVRU were similar between the three groups with no significant difference. These baseline parameters were also similar between subgroups A1, B1, and $\mathrm{C} 1$ and subgroups A2, B2, and C2, with no significant difference.

Table 2 summarizes data pertaining to the perioperative parameters. The mean operative time and mean hemoglobin concentration was significantly higher among patients of group $\mathrm{C}$ compared with groups $\mathrm{A}$ and $\mathrm{B}$ with no significant difference between group $\mathrm{A}$ and $\mathrm{B}(P=0.91$ and $P=0.20)$. The number of patients in whom postoperative irrigation was instituted, amount of fluid used for postoperative irrigation, duration of postoperative irrigation, and duration of catheterization were significantly lower for the patients of group $C$ compared with groups $\mathrm{A}$ and $\mathrm{B}$, with no significant difference between groups $\mathrm{A}$ and $\mathrm{B}(P=0.68, P=0.58, P=0.51$, and $P=0.24$, respectively). Similar statistical differences were found among the patients of subgroup A1, B1, and C1 except for the mean hemoglobin concentration, which did not reveal significant difference among the subgroup patients. Among the subgroups A2, B2, and C2 patients, statistical differences similar to group analysis were found except for the mean amount of irrigation fluid used intraoperatively, which was significantly lower among subgroup C2 patients compared with $\mathrm{A} 2$ and $\mathrm{B} 2$ patients.

Tables 3, 3a, and 3b summarize data pertaining to the follow-up parameters. The follow-up data at 1,3, 6, and 12 months for groups A, B, and C were available for 60, 60, 59, and $57 ; 57,57,55$, and 52; 58, 57, 57, and 55 patients, respectively. All the groups achieved significant improvement





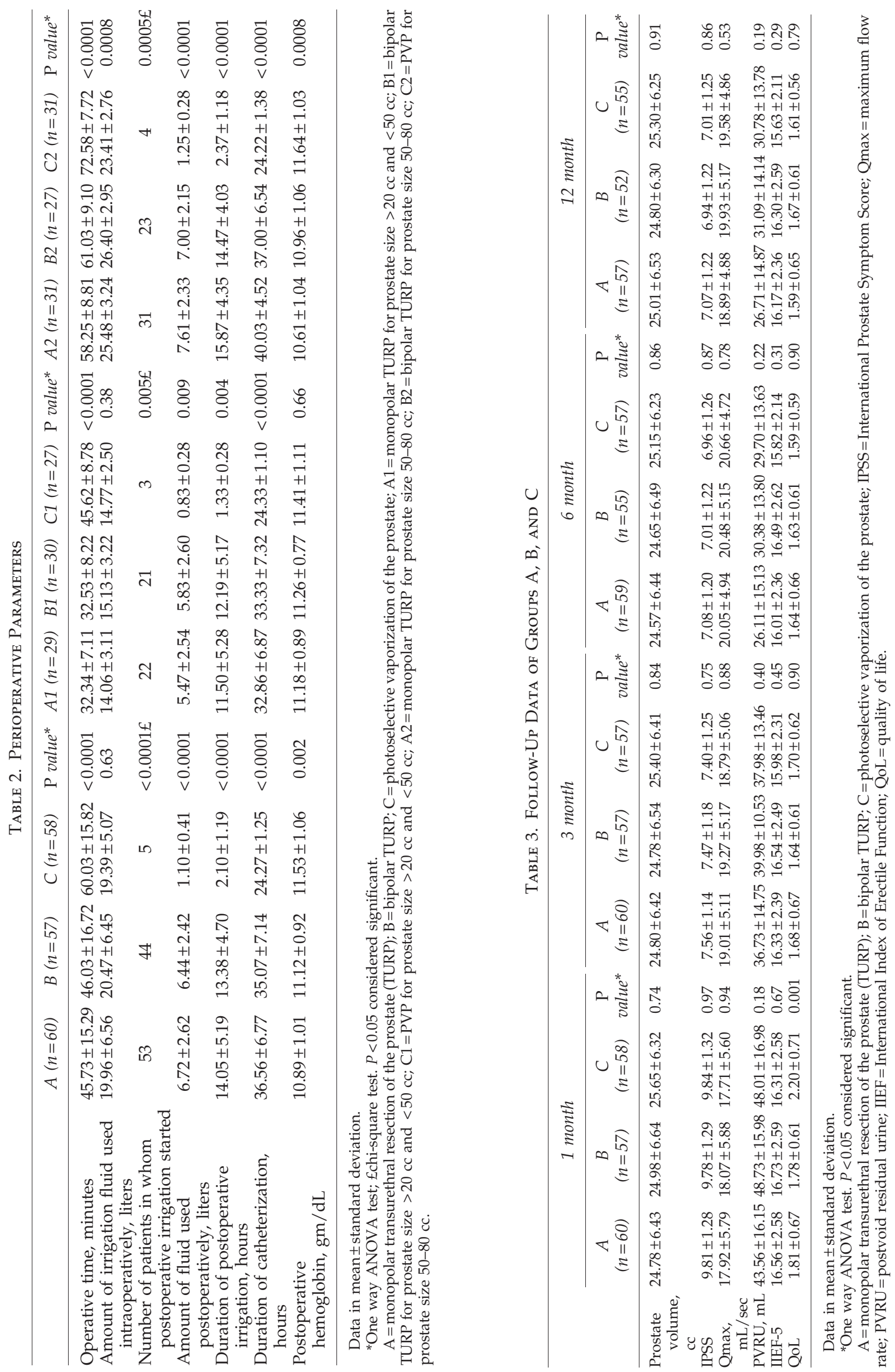

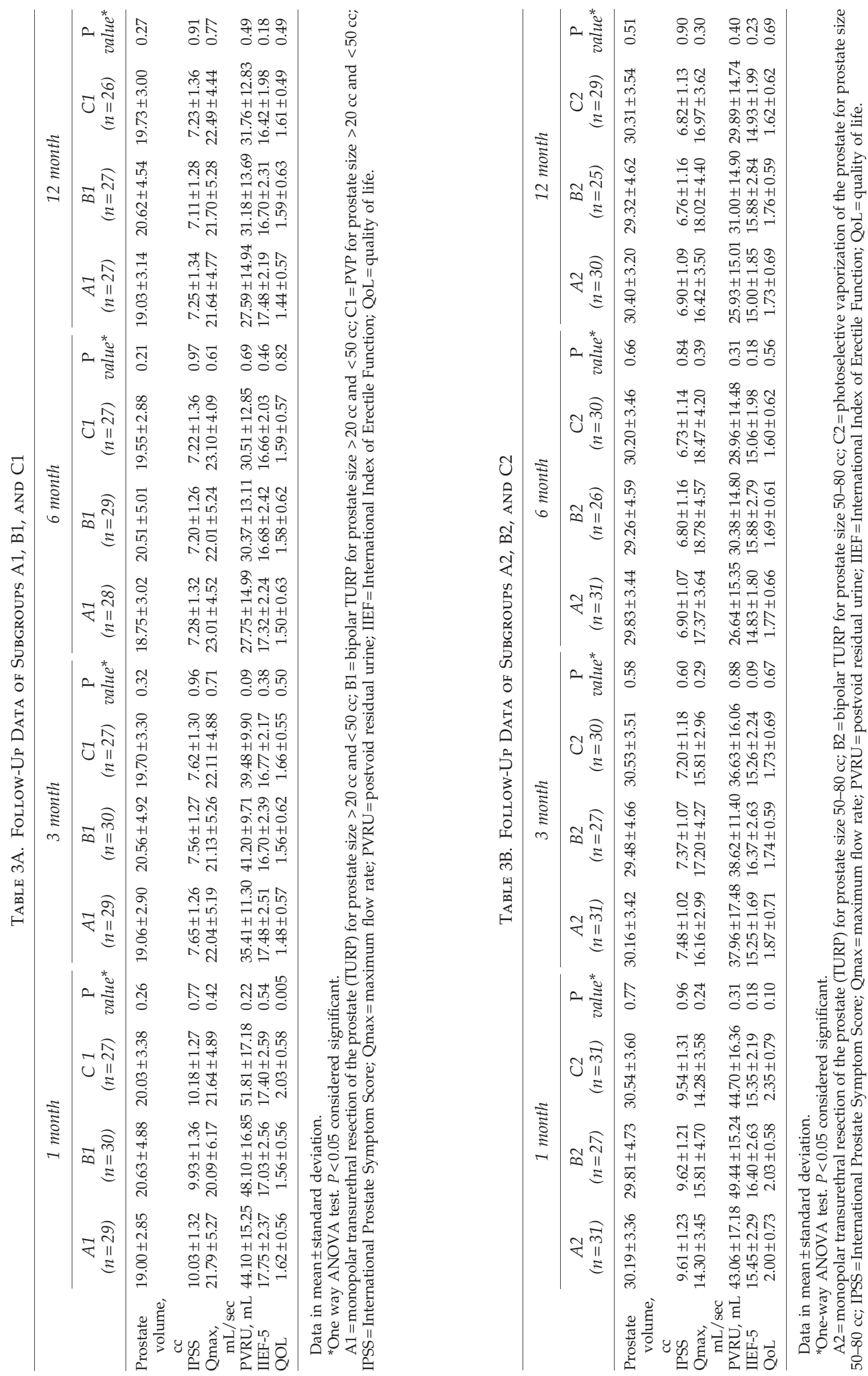
in the IPSS score, QoL score, prostate volume, PVRU, and Qmax at each follow-up visit except for the QoL score at 1month follow up, which was significantly poor among the group $C$ patients $(P=0.001)$. The overall IPSS score decreased by $65.86 \%, 64.87 \%$, and $65.03 \%$, QOL score decreased by $57.90 \%, 53.08 \%$, and $55.27 \%$, prostate volume decreased by $52.08 \%, 50.65 \%$, and $52.07 \%$, Qmax increased by $169.85 \%, 182.69 \%$, and $193.11 \%$, PVRU decreased by $80.81 \%, 79.05 \%$, and $78.52 \%$ at 12 month follow-up in groups A, B, and C, respectively. All groups had shown some decline in the mean IIEF5 score at 12-month follow-up with no significant difference between the groups. The results of subgroups 1 and 2 also revealed similar findings as far as efficacy parameters were concerned.

Table 4 summarizes complication data classified as per the Clavien-Dindo classification of surgical complications. ${ }^{14}$ The overall complication rates were not significantly different between groups and subgroups. The blood transfusion rate and clot retention necessitating intervention were significantly higher among patients of group A compared with group $C(P=0.01)$. Blood transfusion and clot retention were reported in one and two group B patients, respectively, but did not reach a significant level compared with either of groups $\mathrm{A}(P=0.06$ and $P=0.27)$ and $\mathrm{C}(P=1.00$ and $P=0.49$ ). The failed catheter-free trial was reported in four, three, and five patients of group A, B, and C, respectively. Retrial given after 5 days was successful in all 12 patients. None of the complications had shown a significant difference between subgroups A1 vs B1 vs C1. As far as subgroups A2, B2, and C2 were concerned, blood transfusion rate and clot retention necessitating intervention were significantly higher among patients of subgroup A2 compared with subgroup $\mathrm{C} 2$; these complications were intermediate in subgroup B2, which did not reached significant level compared with either subgroup A2 and C2. The complication rates were not different among the patients operated on by the three consultants.

\section{Discussion}

Various modalities of surgical therapy for BPE are available at present, for which long-term data are available for conventional TURP. Perioperative complications are reduced in contemporary studies compared with older ones, such as transfusion rate $(0.4 \%$ vs $7.1 \%)$, TUR syndrome $(0.0 \%$ vs $1.1 \%)$, clot retention ( $2 \%$ vs $5 \%$ ), and urinary tract infection $(1.7 \%$ vs $8.2 \%){ }^{3}$ These improvements were supposed to be because of technologic advancement and better training facilities. The overall morbidity $(11.1 \%$ vs $18 \%)$ and mortality $(0.1 \%$ vs $0.25 \%$ ) rates were also lower in the contemporary series. $3,4,15$

Bipolar TURP and PVP emerged as an alternative to monopolar TURP; several studies have shown their equivalent efficacy with up to 5 years of follow-up. ${ }^{5,6,9-11,16,17}$ Our data support these findings: Efficacy of all the three study groups was comparable up to 12-month follow-up. In our study, all three study groups showed some decline in IIEF-5 score in follow-up visits, which could be explained by the high incidence of preoperative diabetes and preoperative IIEF- $5<21$ in our study population. Only seven, seven, and six patients in groups A, B, and C, respectively, had an IIEF5 score $\geq 21$. There is evidence in the literature supporting the argument that diabetes and poor preoperative IIEF

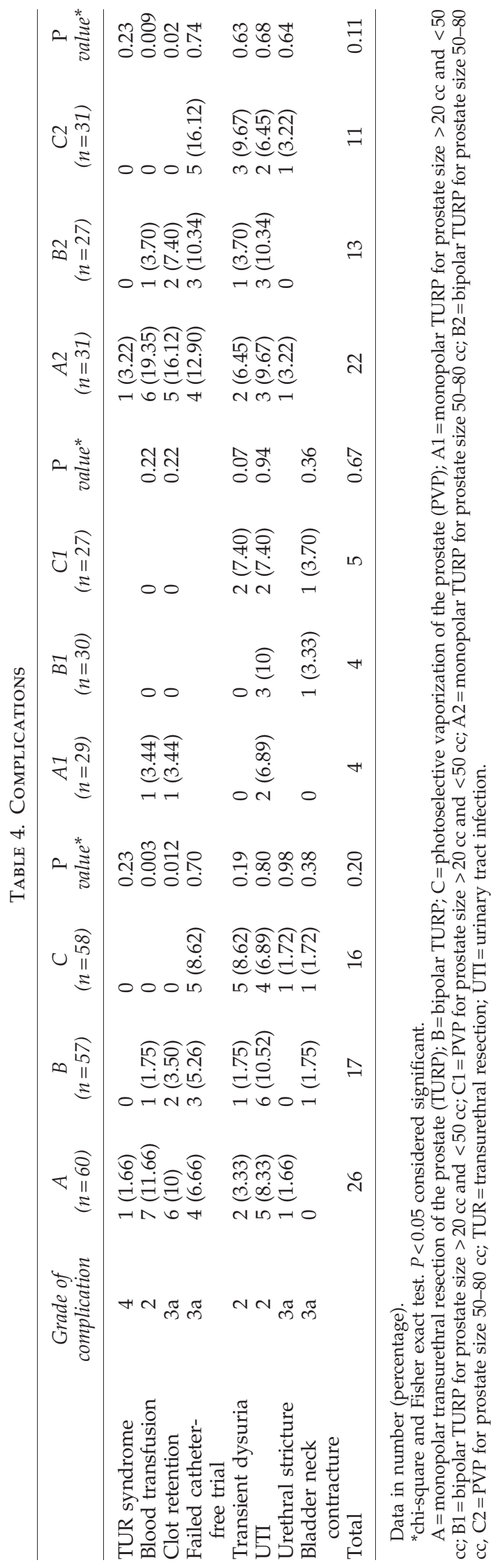




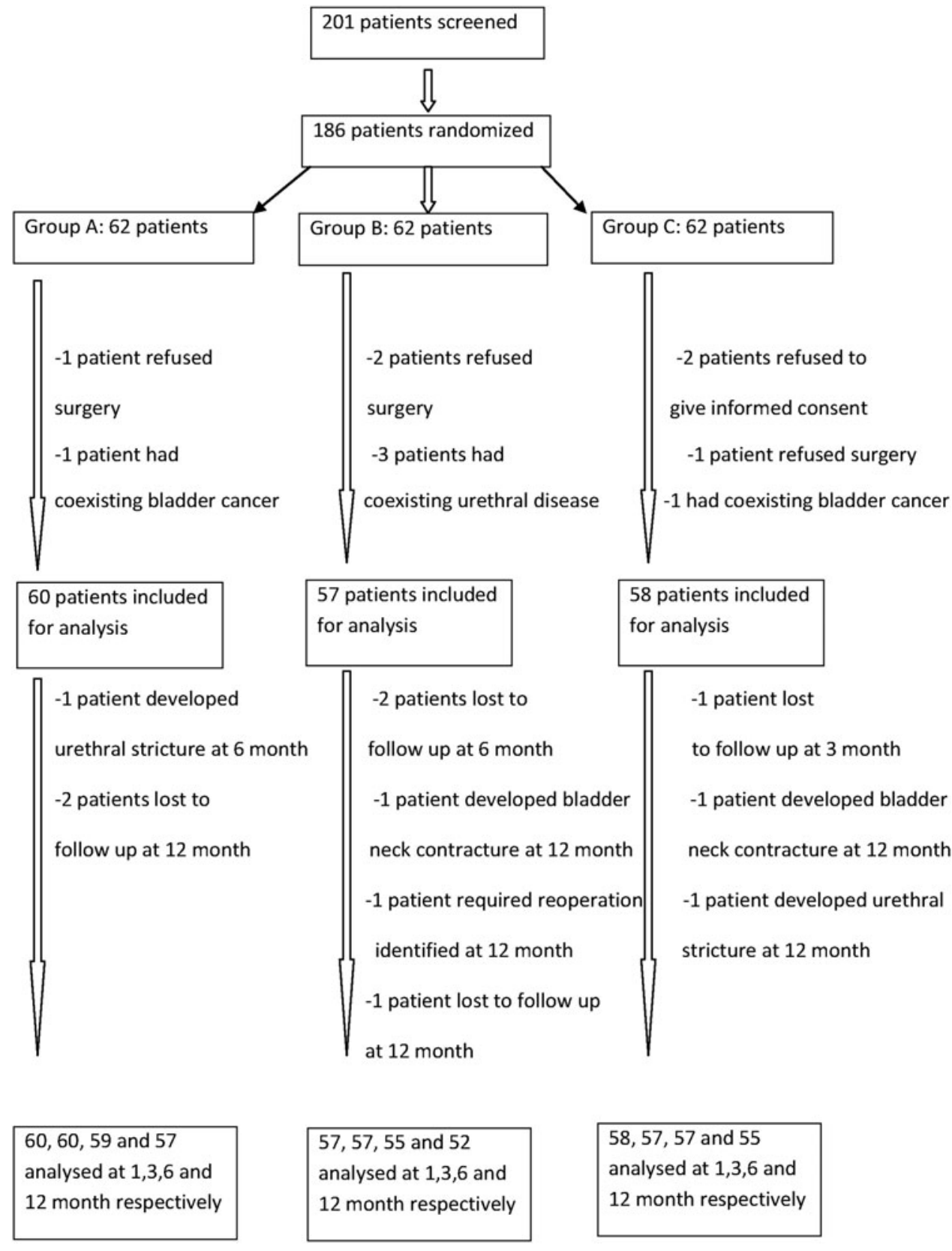

FIG. 1. Allocation and dispersion of patients. score are independent risk factors for postoperative erectile dysfunction. ${ }^{18-20}$

Although some authors reported lower operative duration, fall in hemoglobin, blood transfusion, and clot retention rates for bipolar TURP, 6,16 a meta-analysis of head to head comparison of monopolar and bipolar TURP reported similar operative duration and blood transfusion rates. ${ }^{21}$ Lesser blood loss during bipolar TURP might probably be because of better peroperative quality resulting from less adherence of tissue, better cut capacity, and deeper depth of coagulation. ${ }^{21-23}$ In our study, we observed comparable operative duration, and although the fall in hemoglobin, blood transfusion, and clot retention rates were lower in the bipolar TURP group, it did not reach a significant level compared with the monopolar TURP group. The risk of TUR syndrome was eliminated by bipolar resection of the prostate in saline, even in patients with a large prostate that requires a long duration of operation; these results were safely replicated, even in the hands of residents. ${ }^{17,24,25}$ The rate of TUR syndrome was also reduced with monopolar TURP in the contemporary series. ${ }^{3}$ In our study, TUR syndrome was not significantly different between the groups.

Regarding the duration of postoperative irrigation and duration of catheterization, the majority of the literature is in favor of bipolar TURP compared with monopolar TURP, ${ }^{16,21,26}$ but there are some studies that have shown similar duration of catheterization between monopolar and bipolar TURP..$^{27,28}$ In our study, postoperative irrigation was stopped by the resident on duty depending on the color of the urine, and the catheter was removed 24 hours after the irrigation, if started, was stopped. The decision to start postoperative irrigation was taken by the operative surgeon; blinding for this decision might have a different result. 
Authors reporting results of PVP concluded that it had a significantly lesser fall in hemoglobin and serum sodium levels, capsular perforations, blood transfusion and clot retention rates and also was associated with shorter catheterization time and hospital stay. ${ }^{9-11,13}$ In a prospective, nonrandomized two-center study involving 269 PVP and 127 TURP patients, Ruszat and associates ${ }^{9}$ observed that PVP was favorable in terms of the rate of intraoperative bleeding ( $3 \%$ vs $11 \%$ ), blood transfusions ( $0 \%$ vs $5.5 \%$ ), capsule perforations $(0.4 \%$ vs $6.3 \%)$, postoperative clot retention $(0.4 \%$ vs $3.9 \%)$, and hospitalization time (4.0 vs 5.0 days; $P=0.001$ ) with comparable efficacy at 24-month follow-up. In two recent meta-analyses, it was observed that compared with TURP, PVP, although favorable in terms of perioperative safety, is associated with longer operative time and higher risk of reoperation. ${ }^{29,30}$ Our results of PVP also support the literature regarding perioperative safety parameters, but the risk of reoperation was not significantly different compared with monopolar and bipolar TURP at 12month follow-up.

Results of subgroup analysis were similar to group analysis except (1) the fall in hemoglobin level was less in C1, but it did not reach a significant level compared with A1 and B1; (2) the amount of irrigation fluid used intraoperatively was significantly less in subgroup C2 compared with A2 and B2; (3) complications were comparable among subgroups A1, B1, and $\mathrm{C} 1$.

The potential limitation of the study is exclusion of patients with a prostate size $>80 \mathrm{cc}$. The other limitation is 12 -month follow-up, so comment on long-term efficacy cannot be made.

\section{Conclusion}

Monopolar TURP, bipolar TURP, and PVP are equally efficacious at 12-month follow-up. PVP has added advantages of lesser blood loss, lesser need for blood transfusion (especially for prostate volume 50-80 cc), and shorter catheterization time.

\section{Disclosure Statement}

No competing financial interests exist.

\section{References}

1. Lowe FC. Goals for benign prostatic hyperplasia therapy. Urology 2002;59(suppl 1):1-2.

2. Yu X, Elliott SP, Wilt TJ, McBean AM. Practice patterns in benign prostatic hyperplasia surgical therapy: The dramatic increase in minimally invasive technologies. J Urol 2008;180:241-245.

3. Rassweiler J, Teber D, Kuntz R, Hofmann R. Complications of transurethral resection of the prostate (TURP) -incidence, management, and prevention. Eur Urol 2006;50:969-980.

4. Reich O, Gratzke C, Bachmann A, et al; Urology Section of the Bavarian Working Group for Quality Assurance. Morbidity, mortality and early outcome of transurethral resection of the prostate: A prospective multicenter evaluation of 10,654 patients. J Urol 2008;180:246-249.

5. Ho HS, Cheng CW. Bipolar transurethral resection of prostate: A new reference standard? Curr Opin Urol 2008;18:50-55.

6. Fagerström T, Nyman CR, Hahn RG. Bipolar transurethral resection of the prostate causes less bleeding than the monopolar technique: A single-centre randomized trial of 202 patients. BJU Int 2010;105:1560-1564.

7. Yoon CJ, Kim JY, Moon KH, et al. Transurethral resection of the prostate with a bipolar tissue management system compared to conventional monopolar resectoscope: Oneyear outcome. Yonsei Med J 2006;47:715-720.

8. Kwon JS, Lee JW, Lee SW, et al. Comparison of effectiveness of monopolar and bipolar transurethral resection of the prostate and open prostatectomy in large benign prostatic hyperplasia. Korean J Urol 2011;52:269-273.

9. Ruszat R, Wyler SF, Seitz M, et al. Comparison of potassiumtitanyl-phosphate laser vaporization of the prostate and transurethral resection of the prostate: Update of a prospective non-randomized two-centre study. BJU Int 2008;102:14321439.

10. Bouchier-Hayes DM, Van Appledorn S, Bugeja P, et al. A randomized trial of photoselective vaporization of the prostate using the $80-\mathrm{W}$ potassium-titanyl-phosphate laser vs transurethral prostatectomy, with a 1-year follow-up. BJU Int 2010;105:964-969.

11. Mohanty NK, Vasudeva P, Kumar A, et al. Photoselective vaporization of prostate vs. transurethral resection of prostate: A prospective, randomized study with one year followup. Indian J Urol 2012;28:307-312.

12. Ruszat R, Wyler S, Forster T, et al. Safety and effectiveness of photoselective vaporization of the prostate (PVP) in patients on ongoing oral anticoagulation. Eur Urol 2007;51:1031-1041.

13. Bachmann A, Schürch L, Ruszat R, et al. Photoselective vaporization (PVP) versus transurethral resection of the prostate (TURP): A prospective bi-centre study of perioperative morbidity and early functional outcome. Eur Urol 2005; 48:965-971.

14. Dindo D, Demartines N, Clavien PA. Classification of surgical complications: A new proposal with evaluation in a cohort of 6336 patients and results of a survey. Ann Surg 2004;240:205-213.

15. Mebust WK, Holtgrewe HL, Cockett AT, et al. Transurethral prostatectomy: Immediate and postoperative complications. Cooperative study of 13 participating institutions evaluating 3,885 patients. J Urol 1989;141:243-247.

16. Xie CY, Zhu GB, Wang XH, Liu XB. Five-year follow-up results of a randomized controlled trial comparing bipolar plasmakinetic and monopolar transurethral resection of the prostate. Yonsei Med J 2012;53:734-741.

17. Chen Q, Zhang L, Liu YJ, et al. Bipolar transurethral resection in saline system versus traditional monopolar resection system in treating large-volume benign prostatic hyperplasia. Urol Int 2009;83:55-59.

18. Taher A. Erectile dysfunction after transurethral resection of the prostate: Incidence and risk factors. World J Urol 2004;22:457-460.

19. Akman T, Binbay M, Tekinarslan E, et al. Effects of bipolar and monopolar transurethral resection of the prostate on urinary and erectile function: A prospective randomized comparative study. BJU Int 2013;111:129-136.

20. Kumar A, Vasudeva P, Kumar N, et al. Evaluation of the effect of photoselective vaporization of the prostate on sexual function in a prospective study: A single center experience of 150 patients. J Endourol 2012. Epub ahead of print.

21. Ubee SS, Philip J, Nair M. Bipolar technology for transurethral prostatectomy. Expert Rev Med Devices 2011;8:149154.

22. Huang $\mathrm{X}$, Wang $\mathrm{L}$, Wang $\mathrm{XH}$, et al. Bipolar transurethral resection of the prostate causes deeper coagulation depth 
and less bleeding than monopolar transurethral prostatectomy. Urology 2012;80:1116-1120.

23. Abascal Junquera JM, Cecchini Rosell L, Salvador Lacambra $\mathrm{C}$, et al. [Bipolar versus monopolar transurethral resection of the prostate: Peroperative analysis of the results.] (Spa) Actas Urol Esp 2006;30:661-666.

24. Issa MM, Young MR, Bullock AR, et al. Dilutional hyponatremia of TURP syndrome: A historical event in the 21st century. Urology 2004;64:298-301.

25. Michielsen DP, Coomans D, Braeckman JG, Umbrain V. Bipolar transurethral resection in saline: The solution to avoid hyponatraemia and transurethral resection syndrome. Scand J Urol Nephrol 2010;44:228-235.

26. Kong $\mathrm{CH}$, Ibrahim MF, Zainuddin ZM. A prospective, randomized clinical trial comparing bipolar plasma kinetic resection of the prostate versus conventional monopolar transurethral resection of the prostate in the treatment of benign prostatic hyperplasia. Ann Saudi Med 2009;29:429432 .

27. Engeler DS, Schwab C, Neyer M, et al. Bipolar versus monopolar TURP: A prospective controlled study at two urology centers. Prostate Cancer Prostatic Dis 2010;13:285291.

28. Neyer M, Reissigl A, Schwab C, et al. Bipolar versus monopolar transurethral resection of the prostate: Results of a comparative, prospective bicenter study-perioperative outcome and long-term efficacy. Urol Int 2013;90:62-67.

29. Zhang X, Geng J, Zheng J, et al. Photoselective vaporization versus transurethral resection of the prostate for benign prostatic hyperplasia: A meta-analysis. J Endourol 2012;26: 1109-1117.
30. Teng J, Zhang D, Li Y, et al. Photoselective vaporization with the green light laser vs transurethral resection of the prostate for treating benign prostate hyperplasia: A systematic review and meta-analysis. BJU Int 2013;111:312-323.

Address correspondence to: Anup Kumar, MD, MCh

Department of Urology VMMC and Safdarjung Hospital

New Delhi 110029

India

E-mail: anup_14k@yahoo.com

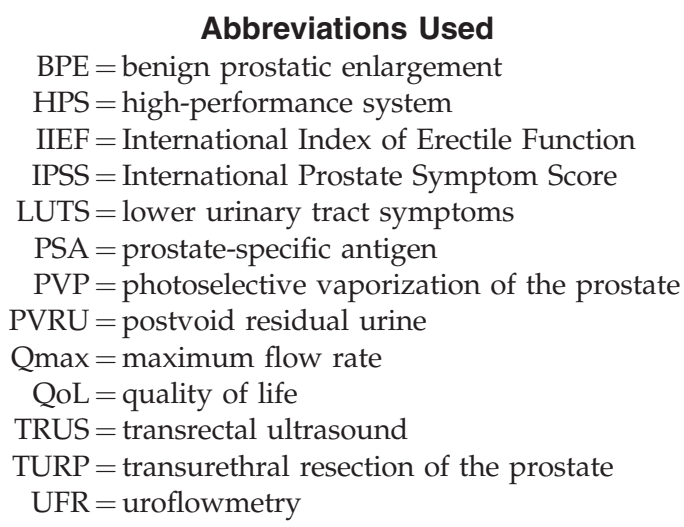

